Aus der Chirurgischen Abteilung des Krankenhauses Bethanien in Berlin. (Chefarzt: Prof. Dr. Martens.)

\title{
Ein Fall von spontaner Uterusruptur in
} der Schwangerschaft. $\left.{ }^{1}\right)$

Von Dr. Fritz Sieber, Assistenzarzt.

Uterusrupturen können in allen Stadien der Gravidität vorkommen; sic sind bisher in allen Schwangerschaftsmonaten, abgesehen vom erstenMonate, beobachtet und beschriebenworden. Immerhin sind sie jedoch, namentlich Fälle von reiner spontaner Ruptur, wie ich in folgendem einen boschreiben möchte, so "solten, daß es sich verlohnen dürfte, jeden einzelnen in der Literatur niederzulegen.

Am 22. Juni 1910 wurde in das Krankenhaus Bethanien eine 23. jähige Frau in moribundem Zustande eingeliefert. Sie hatte bisher zwei Partus durchgemacht und war jetzt in fünften Monate gravide. Nach bisherigem vollkommenen Wollbefinden setzten plötzlich vor $1^{1}$ '́ Tagen lieftige kolikartige Schmerzen ein, die durch den ganzen Leib zogen und dauend anhielten. Der hinzugezogene Arzt konstatier te starke Druckempfindlichkeit des Leibes, die eine genaue bimanuelle Untersuchung ilını nicht ermöglichte; es bestand kein Fieber, innerlich

1) Denronstriert in der Freien Vereinigung der Chirurgen Berlino anı 8. Mai 1911. (Diskussion S. 1720.) 
ließ sich ein leicht geöffneter Muttermund sowie geringe Blutung feststellen, sodaB der Axzt die Diagnose auf beginnenden Abort stellte. Trotzdem er angeordnet hatte, bei eintretender Verschlimmerung sofor $t$ benachrich tigt zu werden, wurde er erst nach etwa 24 Stunden wieder gi holt, fand dis Frau fast moribund und ordnete ihre sofortige Uebelführung ins Krankenhaus an, wo sie aber auch erst einige Stunden danach mit der Diagnose Peritonitis eingeliefert wurde. Blasenwasser war in del $\mathbf{Z}$ wischenzeit nicht abgegangen, auch Blut hatte sich aus der Scheide nur sehr wenig entleert. Trotzdem machte die Frau den Eindruck einer vollkommen Ausgebluteten. Das Gesicht war wachsbleich, eingefallen, die Gesichtszüge ängstlich, die Stixn mit kaltem Schweiß bedeckt, die Extremitäten kalt, der Puls nicht fühl- und zählbar, die Herztöne außer ordentlich leise, die Atmung oberflächlich, stark beschleunigt, mit deutlich zunehmendem Lufthunger. Der Bauch war stalk aufgetrieben, gab lauten tympanitischen Perkussionsschall. Die Bauchdecken waren ziemlich weich. Etwas unterhalb des Nabels fühlte man eine größere Resistenz, Kindsteile waren deutlich unterhalb und rechts vom Nabel fühlbar. Der Muttermund zeigte sich für eine Fingerkuppe durchgängig.

Differentialdiagnostisch kam für uns in erster linie eine Uterusruptur in Frage, wenn man annahm, daß die unterhalb des Nabels gefühlte große Resistenz dem Uterus entspräche, weniger wahrscheinlich war eine geplatzte Extrauteringravidität; doch konnte eine sichere Diagnose wegen des aufgetriebenen Leibes, der eine genaue Palpation nicht zuließ, sowie wegen des elenden Allgemeinbefindens nicht gestellt werden. An einen operativen Eingriff war mit Rücksicht auf den moribunden Zustand der Patientin nicht mehr zu denken. Trotz Herzexzitantien und Kochsalzinfusionen, die wir ausnahmsweise in diesem Falle als letztes Mittel versuchten, sonst aber in solchen Fällen auch erst wälirend oder nach der Operation anzuwenden pflegen, starb die Frau etwa eine Stunde nach der Aufnahme.

Bei der am nächsten Tage vorgenommenen Sektion fanden sich über zwei Liter teils flüssiges, teils geronnenes Blut in der Bauchhöhle. Die Serosa der etwas geblähten Darmschlingen zeigte stellenweise einen ganz zarten, fibrinösen Belag. Der im füntten Monat gravide Uterus weist einen großen, über die Höhe des Fundus verlaufenden, die ganze Fundus breite einnehmenden Querriß auf, der mitten durch die Plazentarstelle hindurchgeht. Durch diesen Riß ist nicht nur der $27 \mathrm{~cm}$ lange Foet hindurchgetreten und liegt nun innerhalb der unversehrten Eihüllen in der freien Bauchhöhle vor dem Uterus, sondern auch die Plazenta hat sich durch den RiB zu etwa 3/4 ihrer Masse hindurcligepreßt, soda $B$ nur noch der vierte Teil von ihr ins Uteruslumen hineinragt. Scheide und außere Genitalien sind vollkommen intakt, ohne jede Spur einer Verletzung, auch die Sektion der übrigen Organe ergibt normalen Befund.

Wie schon eingangs erwähnt, können Uterusrupturen in allen Schwangerschaftsstadien vorkommen, nur jm ersten Schwangerschaftsmonate ist anscheinend bisher noch keine beobachtet worden. $\mathrm{Bais} \mathrm{ch}^{1}$ ), der aus der Literatur 78 Fälle von Uterusruptur in der Gravidität zusamnienstellte, fand folgende Verteilung auf die einzelnen Schwangerschaftsmonate: Es ereigneten sich im 2. Monate 2 Fälle, im 3. 7, im 4. 12, im 5 . 10 , in 6.11 , im 7.7 , im 8.6, im 9.6 , im 10.12 und unbestinimt 5 Fälle.

Was das Zustandekommen der Ruptur anbelangt, so finden wir in mindestens dem vierten Teile aller Fälle als Ursache ein Traunia angegeben, und zwar meist ein indirekt wirkendes Trauma, wie Fall auf die Fïße, auf Rücken und Gesäß etc. Auch das Heben einer schweren Last, starkes Pressen beim Stuhlgange ist in einzelnen Fällen angeschuldigt worden. In allen übrigen Fällen läßt sich dagegen von irgendeinem Trauma nichts feststellen. Auch in unserem Falle ist von einem solchen keine Rede, ebenso läßt sich hier eine direkte Verletzung durch kriminelle Abtreibungsversuche vollkommen ausschließen; schon der Sitz des Risses spricht bei den vollständig intakten Eihüllen mit Sicherheit dagegen. In einen weiteren Teile der Fälle fand sich als Ursache der Ruptur eine gröbere pathologischr Veränderung des Uterus, z. B. myomatöse und karzinomatöss: Neubildung, oder auch eine angeborene Mißbildung, wie Schwangerschaft im rudimentären Nebeuhorn. Von allen diesen leicht greifbaren Ursachen ist, wie in einem größeren Teile der bisher beschriebenen Fälle, auch in unserem Falle nicht die Rede; hier handelt es sich vielmehr u m spontane, anscheinend ohne nachweisbare Ursache auftretende Rupturen.

Man hat natürlich auch in solchen Fällen nach einer Ursache gesucht, da nicht ohne weiteres anzunehmen ist, daß ein vollkommen normaler Uterus ohne jede Veranlassung in der Schwangerschaft zur Ruptur kommen kann. Als wichtig haben sich nun in dieser Beziehung die anamnestischen Erhebungen, namentlich über den Verlauf eventueller früherer Geburten erwiesen. Denn in einem großen Teile der bisher unerklärlichen Fälle hat man eine während einer früheren Geburt erlittene Verletzung nachweisen können, die zur Narbenbildung innerhalb der Uterusmuskulatur Veranlassung gab. Als solche narbenbildende und dadurch zu. späteren Uterusrupturen disponierende Momente hat man neben den in dieser Beziehung wichtigsten Kaiserschnitten noch tiefe Zervixrisse und -schnitte, Verletzungen bei Zangengeburten, durch Kurettement und durch manuclle Plazentarlösungen gefunden. Alle solche früheren Geburtstraumen können zu einer Narbenbildung im Bereiche der Uterusmuskulatur führen und bedingen dadurch eine Verdünnung der Gebärnutterwand und eine erhebliche Herabsetzung ihrer Widerstandsfähigkeit im Verlaufe späterer Schwangerschaften. Und wenn auch erfahrungsgemäß das Narbengewebe allmählich wieder durch neu sich bildendes Muskelgewebe ersetzt zu werden pflegt, so bildet doch die betreffende Stelle für längere Zeit einen Locus minoris resistentiae in späteren Schwangerschaften, zumal wenn sich die Plazenta im Bereiche der narbig veränderten Stelle ansiedelt. Dementsprechend sind auch solche spontanen Uterusrupturen während der Gravidität fast nur bei Mehrgebärenden beschrieben worden. Auch in unserem Falle ließ sich noch nachträglich feststellen, daß bei den zwei früher erfolgten Partus, und zwar entweder bei einem oder bei allen beiden - Genaueres war ananinestisch hierüber nicht mehr zu eruieren -, die manuelle Plazentarlösung vorgenommen worden war. Nun konnte allerdings weder makroskopisch noch durch die mikroskopische Untersuchung an der Rißstelle Narbengewebe gefunden werden, dagegen zeigt sich die. Uteruswand im Bereiche des Risses verdünnt, das Plazentargewe be tief in die Wandung eingewuchert. Auch von sonstigen degenerativen Veränderungen der Muscularis, wie nian sie in nur vereinzelten Fällen gefunden hat und als Ursache der Ruptur angesehen wissen will, wie z. $B$. fettige Entartung, liyaline Degeneration, Mangel an elastischen Fasern, kleinzellige Infiltration, war mikroskopisch nichts festzustellen. Und iibereinstimmend damit hat auch in der übergroßen Mehrzahl der früher beschriebenen Fälle die mikroskopische Untersuchung bisher noch keine positiven Resultate zu erbringen vermocht; auch der Nachweis von Narbengewebe ist mit voller Sicherheit noch selten gelungen.

Wir müssen uns also damit begnügen, mit hoher Wahrscheinlichkeit anzunehmen, daß in unserem Falle das bei Gelegenheit der manuellen Plazentarlösung erlittene frühere Geburtstrauma narbige Veränderungen der Uterusmuskulatur bedingte, dic die Widerstandsfähigkeit der Gebärmutterwandung in einem Maße herabsetzten, daß es bei einer während einer späteren Schwangerschaft erfolgten Plazentaransiedlung an dieser Stelle mit tiefer Einwucherung der Zotten in die verdïnnte Wand ohne irgendwelche erkennbare äußere Einwirkung zu einer Ruptur in so frïhem Schwangerschaftsstadium kommen konnte.

1) Ba isch, Zentralblatt, für Gynäkologie 1904, No. 28. 